\title{
La construcción de la identidad personal y profesional de los deportistas de alto nivel*
}

\author{
David López-Aguilar \\ Pedro Ricardo Âlvarez-Pérez \\ Universidad de La Laguna. España. \\ dlopez@ull.edu.es \\ palvarez@ull.edu.es
}

Recibido: 27/10/2017

Aceptado: 4/12/2018

Publicado: 15/7/2019

\section{Resumen}

La retirada de la práctica deportiva constituye una de las fases más complejas en la trayectoria vital de los deportistas de alto nivel, y requiere de una adecuada preparación y planificación previas. La definición del proyecto profesional y vital puede ayudar a los deportistas a afrontar este proceso antes, durante y después de la retirada de su actividad. En relación con esta problemática, el objetivo central del presente estudio fue analizar la influencia de distintos factores asociados al desarrollo profesional y vital de deportistas españoles de alto nivel. Para la recogida de datos se empleó una entrevista semiestructurada, que se organizó en torno a cinco dimensiones básicas: competición deportiva de alto nivel (I); fase exploratoria, conocimiento de sí mismo (II); fase exploratoria, conocimiento del entorno (III); fase de definición de las metas profesionales (IV), y fase de toma de decisiones vocacionales y profesionales $(\mathrm{V})$. La muestra estuvo compuesta por un total de 16 deportistas de alto nivel acreditados por el Consejo Superior de Deportes. A partir de los resultados obtenidos, cabe destacar que los participantes en el estudio eran conscientes de la importancia que tenía la formación académica y el diseño de un proyecto personal y de vida sobre su futuro desarrollo profesional.

Palabras clave: desarrollo profesional; proyecto personal; deportistas de élite; autoeficacia; expectativas de resultado

\section{Resum. La construcció de la identitat personal i professional dels esportistes d'alt nivell}

La retirada de la pràctica esportiva constitueix una de les fases més complexes en la trajectòria vital dels esportistes d'alt nivell, y requereix una adequada preparació i planificació prèvies. La definició del projecte professional i vital pot ajudar els esportistes a afrontar aquest

* Este trabajo forma parte de la tesis doctoral titulada El desarrollo vocacional y la construcción del proyecto profesional y vital en deportistas de alto nivel, elaborada por David López Aguilar (<http://dlopez.webs.ull.es>) y dirigida por Pedro Ricardo Álvarez Pérez (<http://palvarez.webs. ull.es $>$ ). Este trabajo ha contado con la financiación del Plan Nacional de Formación de Profesorado Universitario del Ministerio de Educación, Cultura y Deportes (FPU-MEC), perteneciente al Programa Estatal de Promoción del Talento y su Empleabilidad, en el marco del Plan Estatal de Investigación Científica y Técnica de Innovación. Ambos autores pertenecen al Grupo Universitario de Formación y Orientación Integrada (GUFOI). 
procés abans, durant i després de la retirada de la seva activitat. En relació amb aquesta problemàtica, l'objectiu central del present estudi va ser analitzar la influència de diferents factors associats al desenvolupament professional i vital d'esportistes espanyols d'alt nivell. Per fer la recollida de dades es va emprar una entrevista semiestructurada, que es va organitzar entorn a cinc dimensions bàsiques: competició esportiva d'alt nivell (I); fase exploratòria, coneixement de si mateix (II); fase exploratòria, coneixement de l'entorn (III); fase de definició de les metes professionals (IV), i fase de presa de decisions vocacionals i professionals (V). La mostra va estar composta per un total de 16 esportistes d'alt nivell acreditats pel Consell Superior d'Esports. A partir dels resultats obtinguts, cal destacar que els participants en l'estudi eren conscients de la importància que tenia la formació acadèmica i el disseny d'un projecte personal i de vida sobre el seu futur desenvolupament professional.

Paraules clau: desenvolupament professional; projecte personal; esportistes d'elit; autoeficiència; expectatives de resultats

\title{
Abstract. Construction of the personal and professional identity of high-level athletes
}

Withdrawal from sport is one of the most complex stages in the life trajectories of high-level athletes and requires appropriate preparation and planning. Defining a professional and life project can help athletes cope with this process before, during, and after withdrawing from sport. In this regard, the main aim of this study was to analyze the influence of different factors associated with the professional and personal development of high-level Spanish athletes. Data were collected by means of a semi-structured interview organized into five basic dimensions: (I) sports competition; (II) exploratory phase and self-knowledge; (III) exploratory phase and knowledge of the environment; (IV) defining career goals; and (V) vocational and professional decisions. The sample comprised a total of 16 high-level athletes accredited by the Higher Sports Council of Spain. The results show that the athletes were aware of the importance of academic training and designing a personal life project for their future professional development.

Keywords: professional development; personal project; high-level athletes; self-efficacy; outcomes

\author{
Sumario \\ 1. Introducción 4. Conclusiones \\ 2. Método Referencias bibliográficas
}

3. Resultados

\section{Introducción}

Una de las etapas vitales que mayor dificultad y riesgo comporta para el deportista de alto nivel es el de la retirada de su actividad competitiva y la transición hacia el mundo del trabajo (Garcés y Carlin, 2011; Wylleman, Lavallee y Alfermann, 1999). Es una complicada fase en su trayectoria personal, puesto que se ve obligado a abandonar o a simultanear la competición de alto rendimiento para dar paso a nuevos desempeños y nuevos roles vitales. De acuerdo con Kerr y Dacyshyn (2000), es «el momento de dedicar su energía a otras 
actividades como la educación y la planificación de la carrera» (p. 122). De hecho, tras la finalización de la práctica deportiva de alto nivel, el $28 \%$ de los deportistas continúa con su formación y el $21 \%$ busca un empleo (North y Lavallee, 2004).

La retirada deportiva es vivida por muchos deportistas como un complicado momento caracterizado por la pérdida de la identidad personal, la existencia de dificultades emocionales, el encuentro con nuevos sentimientos y emociones, como el aislamiento y la soledad, la frustración, la baja autoestima, la poca satisfacción con la nueva etapa, la escasa cualificación profesional, etc. (Surujlal y Zyl, 2014; González y Torregrosa, 2009; Bruner, Munroe-Chandler y Spink, 2008; Fernández, Stephan y Fouquereau, 2006; North y Lavallee, 2004). De acuerdo con Surujlal y Zyl (2014), muchas de las dificultades encontradas en esta compleja etapa de la vida de los deportistas son atribuidas principalmente al hecho de que, a lo largo de su recorrido vital, no se han preocupado por ir planificando ni preparando un proyecto profesional y vital que los guíe y los oriente en su trayectoria personal. Esta falta de proyección hacia el futuro conduce a una situación en la que atraviesan un largo periodo de transición que va desde la aceptación de la finalización deportiva hasta la adaptación a su nuevo estilo vital (González y Torregrosa, 2009; Surujlal y Zyl, 2014).

En los hallazgos del trabajo realizado por Baillie y Danish (1992) se concluyó que la planificación de un proyecto de vida correlacionaba fuertemente con la satisfacción que encontraba el deportista en las diferentes etapas que atravesaba en su recorrido personal. Efectivamente, los deportistas que a lo largo de su vida habían tenido una actitud planificadora se enfrentaron a menos problemas emocionales, se integraron y se adaptaron con mayor facilidad a su entorno, contaron con estrategias y herramientas para afrontar las dificultades que encontraron en su trayectoria vital y experimentaron una mayor satisfacción respecto a sus vidas (Carlin y Garcés, 2012; Wong, 2010; Warriner y Lavallee, 2008). Por ello, la planificación y la definición de un proyecto profesional y de vida (Alfermann, Stambulova y Zemaityte, 2004) debería ser una preocupación constante y una parte importante en la vida del deportista.

Sin embargo, y a pesar de la importancia que tiene la competencia de la planificación para el deportista de alto nivel, solo una minoría de casos se preocupa por ir preparando un proyecto de vida que lo guíe para el antes, el durante y el después de la retirada de la competición (Carlin y Garcés, 2012; Jodai y Nogawa, 2012; Wong, 2010). En un estudio de revisión realizado por Cecic-Erpic, Wylleman y Zupancic (2004) se encontró que aproximadamente el $45 \%$ de los deportistas no planificaron un proyecto que guiara y orientara su desarrollo vital.

González y Bedoya (2008) y González y Torregrosa (2009) afirman que la respuesta a esta situación pasa por el diseño y la puesta en marcha de programas de orientación cuyo objetivo prioritario es ayudar al deportista de alto nivel a construir planes y proyectos para su desarrollo personal y profesional. 
Concretamente, estos autores sostienen que, a lo largo de la vida, el deportista debería trabajar temas relacionados con la orientación para el desarrollo de la carrera, el asesoramiento académico, la toma de decisiones académicas y profesionales, la búsqueda de empleo, la adquisición de competencias profesionales, etc. En definitiva, se debe «dotar a las personas de las competencias necesarias para identificar, elegir y/o reconducir alternativas formativas y profesionales, acordes a su [...] proyecto vital» (Echeverría, 1997, p. 97).

La orientación, y más concretamente el proyecto profesional y vital, podría resultar una importante herramienta para ayudar al deportista de alto nivel a planificar su plan personal y de vida. El proyecto profesional y vital es una estrategia orientadora con un marcado carácter preventivo y personalizador, en la que los deportistas adquieren y desarrollan competencias transferibles y de adaptabilidad (Taylor y Ogilvie, 1988), reciben apoyo y ayuda durante todo su desarrollo vital (Sarason y Sarason, 1986) y preparan y planifican un plan de acción que los guía y los orienta en todo su recorrido personal (Pearson y Petipas, 1990).

La elaboración de este proyecto surge a partir del continuo cuestionamiento entre las acciones del pasado y las del presente, con la finalidad de proyectarlas hacia el futuro (Rodríguez, Álvarez, Figuera y Rodríguez, 2009; Martínez, 2008). Este ejercicio de construcción del proyecto de vida requiere la clarificación de la imagen de sí mismo, la definición de metas académicas y profesionales, la adquisición de conocimientos, la mejora de ciertas habilidades, etc. (Rodríguez, 1999; Guichard, 1995). Se trata de una experiencia de construcción personal que se produce a lo largo de la trayectoria vital del deportista, en la que se plantean aspectos vinculados a la personalidad, a la situación del entorno, a la manera de relacionarse con el contexto y a lo que se desea y se quiere para el futuro personal (Rodríguez et al., 2009; Romero, 2004; Rodríguez, 1999; Romero, Seco y Lugo, 2015). Como sugieren Patton y McMahon (2006), la definición de este proyecto viene determinada por la relación entre el sistema interpersonal (sexo, edad, personalidad, habilidades, intereses y metas profesionales, etc.), el sistema social (familia, deportistas, entrenadores, etc.) y el sistema macrosocial (normas sociales, situación económica, oportunidades laborales, etc.).

Lent, Brown y Hackett (1994) y Lent, Brown y Hackett (2000) sostienen que, en la elaboración de los proyectos profesionales y vitales, desempeñan un importante papel los conceptos de autoeficacia y las expectativas de resultado. Por una parte, la autoeficacia es la capacidad que tiene el individuo para valorar en qué medida las competencias, los conocimientos y las habilidades que posee le permitirán alcanzar determinados niveles de rendimiento en una acción concreta y, por otra, las expectativas de resultado hacen referencia a lo que espera alcanzar el deportista en una actividad específica. De la relación equilibrada entre estos dos constructos (autoeficacia y expectativas de resultado) surge la definición de intereses profesionales y de metas personales que se sustentan sobre una base realista y que atiende a las características personales, ambientales y situacionales del deportista de alto nivel. 
Precisamente, el propósito principal de este trabajo de investigación ha sido analizar y reflexionar acerca de algunas cuestiones clave relativas a los procesos de desarrollo profesional y de construcción del proyecto de vida de los deportistas de alto nivel del contexto español. De manera más precisa, los objetivos que guiaron el estudio fueron:

- Analizar la importancia y el sentido que tiene el trabajo para los deportistas de alto nivel.

- Analizar la importancia que tienen los procesos formativos en el desarrollo profesional de los deportistas de alto nivel.

- Analizar el conocimiento que tienen los deportistas de alto nivel sobre el mundo profesional.

- Analizar los intereses y las metas profesionales de los deportistas de alto nivel.

- Analizar el estilo de toma de decisiones de los deportistas de alto nivel.

\section{Método}

En este trabajo de investigación se utilizó una metodología cualitativa de corte descriptivo no experimental, basado en la aplicación de la entrevista semiestructurada como técnica de recogida de datos (Del Rincón, Latorre, Arnal y Sans, 1995). El escaso conocimiento sobre los procesos de desarrollo profesional y de construcción del proyecto laboral y vital de los deportistas de alto nivel en el contexto español justifica la necesidad de realizar este tipo de estudios, cuyos hallazgos podrían contribuir a la comprensión de esta realidad y a definir estrategias y mecanismos de intervención desde la práctica educativa y orientadora que ayuden a estos deportistas a definir su identidad vocacional y su proyecto existencial.

\subsection{Participantes}

Los participantes que cumplimentaron la entrevista eran deportistas de alto nivel que previamente participaron en un estudio cuantitativo sobre el proceso de construcción del proyecto profesional y vital. Concretamente, los criterios que se tuvieron en cuenta a la hora de seleccionar a los deportistas que participaron en la entrevista fueron los siguientes:

- Deportistas de alto nivel de diferentes zonas geográficas de España.

- Deportistas de alto nivel pertenecientes a distintas categorías deportivas: deportistas acreditados por el Consejo Superior de Deportes (CSD), por el plan de la Asociación de Deportes Olímpicos (ADO) o por las federaciones incluidas en las selecciones nacionales.

- Deportistas de alto nivel que participaron en competiciones internacionales, nacionales, regionales y/o locales. 
Tabla 1. Características de la muestra participante en la entrevista

\begin{tabular}{|c|c|c|c|c|c|c|}
\hline \multicolumn{7}{|c|}{ Número total de deportistas de alto nivel que cumplimentaron la entrevista: $16(n=16)$} \\
\hline $\begin{array}{c}\text { Etapa } \\
\text { deportiva }\end{array}$ & $\begin{array}{l}\text { Codifi- } \\
\text { cación }\end{array}$ & $\begin{array}{l}\text { Edad } \\
\text { media }\end{array}$ & Género & $\begin{array}{l}\text { Formación } \\
\text { académica }\end{array}$ & $\begin{array}{c}\text { Comunidad } \\
\text { autónoma }\end{array}$ & $\begin{array}{l}\text { Categoría } \\
\text { deportiva }\end{array}$ \\
\hline \multirow[t]{4}{*}{ Iniciación } & EID1 & \multirow{4}{*}{16} & Hombre & Preuniversitaria & Madrid & DAN CSD \\
\hline & EID2 & & Mujer & Preuniversitaria & Navarra & DAN CSD \\
\hline & EID3 & & Hombre & Preuniversitaria & Cáceres & $\begin{array}{l}\text { DAN selección } \\
\text { nacional }\end{array}$ \\
\hline & EID4 & & Hombre & Preuniversitaria & Andalucía & $\begin{array}{l}\text { DAN selección } \\
\text { nacional }\end{array}$ \\
\hline \multirow{4}{*}{$\begin{array}{l}\text { Perfeccio- } \\
\text { namiento }\end{array}$} & EPD1 & \multirow{4}{*}{20,50} & Mujer & Universitaria & Cataluña & DAN CSD \\
\hline & EPD2 & & Hombre & Preuniversitaria & Valencia & DAN CSD \\
\hline & EPD3 & & Hombre & Preuniversitaria & Madrid & DAN ADO \\
\hline & EPD4 & & Hombre & Universitaria & Canarias & $\begin{array}{l}\text { DAN selección } \\
\text { nacional }\end{array}$ \\
\hline \multirow{4}{*}{$\begin{array}{l}\text { Preparación } \\
\text { de la } \\
\text { retirada }\end{array}$} & EPR1 & \multirow{4}{*}{46,25} & Hombre & Preuniversitaria & Cataluña & DAN ADO \\
\hline & EPR2 & & Hombre & Universitaria & Extremadura & $\begin{array}{l}\text { DAN selección } \\
\text { nacional }\end{array}$ \\
\hline & EPR3 & & Hombre & Preuniversitaria & Navarra & DAN CSD \\
\hline & EPR4 & & Hombre & Universitaria & Cataluña & DAN CSD \\
\hline \multirow{4}{*}{$\begin{array}{l}\text { Retirada } \\
\text { deportiva }\end{array}$} & ERD1 & \multirow{4}{*}{33,75} & Mujer & Universitaria & Canarias & DAN CSD \\
\hline & ERD2 & & Hombre & Preuniversitaria & Asturias & DAN CSD \\
\hline & ERD3 & & Mujer & Universitaria & Asturias & DAN CSD \\
\hline & ERD4 & & Hombre & Universitaria & Madrid & DAN CSD \\
\hline
\end{tabular}

Fuente: elaboración propia.

Tal y como se presenta en la tabla 1 , del total de personas identificadas, participaron finalmente $16(n=16)$ deportistas de alto nivel de diferentes zonas geográficas de España, distribuidos de manera equitativa en las diferentes etapas deportivas propuestas por Wylleman y Lavalle (2004).

\subsection{Instrumento de recogida de información}

Corbeta (2003) y Hernández, Fernández-Collado y Baptista (2006) afirman que una de las principales ventajas que tiene la entrevista es que se trata de una técnica más flexible y abierta que otras herramientas de recogida de datos como son el cuestionario o el grupo de discusión. A través de la entrevista, se logra una comunicación y una construcción conjunta de significados respecto a un tema objeto de interés para el investigador.

Para el diseño de la entrevista utilizada en este estudio se realizaron diferentes reuniones de trabajo, en las que colaboraron dos investigadores expertos en la orientación académica y profesional $(n=2)$ y dos especialistas en la metodología de la investigación cualitativa en el área de las ciencias sociales $(n=2)$. En estas reuniones se valoraron aspectos relacionados con el contenido a tratar en la entrevista, el tipo de preguntas a realizar, el posible tratamiento de la infor- 
mación, etc. Otro mecanismo que se empleó en el proceso de confección de la entrevista fue la elaboración de una prueba piloto con dos deportistas de alto nivel $(n=2)$ que reunieron características similares a los participantes en la muestra definitiva. En esta prueba se valoraron aspectos referidos al tiempo de respuesta, al orden de las preguntas, a la pertinencia del contenido, etc.

Asimismo, y de cara a aplicar la entrevista semiestructurada, se valoraron distintas posibilidades teniendo en cuenta especialmente que las personas que participaron en esta prueba pertenecían a diferentes comunidades autónomas de España. Atendiendo a este criterio, se decidió emplear una entrevista telefónica semiestructurada con la finalidad de acceder a deportistas de alto nivel de distintas zonas geográficas. Para valorar alternativas que permitieran realizar llamadas telefónicas y grabarlas digitalmente de manera simultánea, se consultó a expertos del servicio de nuevas tecnologías de la Universidad de La Laguna $(n=2)$. Tras analizar diferentes opciones, finalmente se decidió emplear un programa informático tipo SoftPhone denominado 3CXPhone, dado que, entre sus bondades, permitió realizar llamadas y registrarlas digitalmente de manera ilimitada para posteriormente transcribirlas, analizarlas e interpretarlas.

A partir de este procedimiento se construyó el guión definitivo, que quedó configurado por un total de 19 preguntas que giraron en torno a cinco dimensiones básicas: competición deportiva de alto nivel (I); fase exploratoria, conocimiento de sí mismo (exploración) (II); fase exploratoria, conocimiento del entorno (exploración) (III); fase de definición de las metas profesionales (cristalización) (IV), y fase de toma de decisiones vocacionales y profesionales (especificación) (V).

En el transcurso de la administración de la entrevista se siguieron algunas de las orientaciones y recomendaciones propuestas por Franklin y Ballau (2005), McMillan y Schumacher (2005), Mertens (2005) y Hernández Fernández-Collado y Baptista (2006), con la finalidad de asegurar la confiabilidad, la validez y la objetividad de los datos recogidos:

- Anotación de incidencias o de aspectos que fueron de interés para dar respuesta a los objetivos del estudio.

- Grabación de las sesiones de manera digital para posteriormente ser transcritas verbatim.

- Participación de distintos investigadores en el proceso de codificación de la entrevista, con la finalidad de contrastar la información obtenida.

\subsection{Procedimiento}

Una vez elaborado el guion definitivo, se llevó a cabo la recogida de datos. Concretamente, durante el segundo semestre del año 2015 se contactó con los deportistas de alto nivel que participaron en la entrevista. Para ello se utilizó el siguiente procedimiento:

1. Teniendo en cuenta los criterios de selección, se identificaron, de manera aleatoria, en la base de datos del estudio cuantitativo realizado previamente, 
10 deportistas de la fase de iniciación deportiva, 10 de perfeccionamiento, 10 de preparación de la retirada y 10 deportistas retirados.

2. Se realizó un envío de correo electrónico masivo a los deportistas identificados, con la intención de informarles sobre la finalidad de participar en una entrevista telefónica relacionada con la construcción del proyecto profesional y vital de los deportistas de alto nivel.

3. Los deportistas que accedieron a participar en la entrevista fueron convocados en una hora y en una fecha determinadas previo acuerdo entre deportista e investigador.

4. Los días previos a la entrevista se envió un correo electrónico a modo de recordatorio al deportista de alto nivel, con la finalidad de confirmar la hora y la fecha acordadas.

\subsection{Análisis de la información}

El análisis cualitativo recogido a partir de las entrevistas se realizó con el apoyo metodológico del programa informático Atlas-Ti en su versión 6.0 para el sistema operativo Microsoft Windows 7. Este programa disponía de un potente conjunto de herramientas para analizar información cualitativa en soporte texto, gráfico o vídeo. En esta aplicación se generó una unidad hermenéutica (Hermeneutic Unit) con toda la información (contenido de las entrevistas realizadas), a partir de la cual se identificaron los codes y las variables (outputs) que sirvieron para interpretar la información obtenida (tabla 2). Además, este

Tabla 2. Sistema de codes

\begin{tabular}{|c|c|c|}
\hline N. ${ }^{\circ}$ & Codificación (code) & Objetivo del code \\
\hline 1 & $\mathrm{CL}$ - Conocimiento laboral & $\begin{array}{l}\text { Analizó el conocimiento que tenían los deportistas de alto } \\
\text { nivel sobre el mercado laboral. }\end{array}$ \\
\hline 2 & CT - Centralidad del trabajo & $\begin{array}{l}\text { Analizó la importancia que concedieron los deportistas de } \\
\text { alto nivel a su desarrollo profesional. }\end{array}$ \\
\hline 3 & FT - Finalidad del trabajo & $\begin{array}{l}\text { Valoró la finalidad y el sentido que tenía el trabajo para los } \\
\text { deportistas de alto nivel. }\end{array}$ \\
\hline 4 & $\begin{array}{l}\text { HIPPV - Hitos importantes } \\
\text { en el proyecto profesional } \\
\text { y vital }\end{array}$ & $\begin{array}{l}\text { Identificó posibles hitos importantes, personas relevantes, } \\
\text { aspectos clave, etc. que determinaron la construcción del } \\
\text { proyecto profesional y vital de los deportistas de alto nivel. }\end{array}$ \\
\hline 5 & $\begin{array}{l}\text { OL - Oportunidades labo- } \\
\text { rales }\end{array}$ & $\begin{array}{l}\text { Analizó las oportunidades laborales que tuvieron los depor- } \\
\text { tistas de alto nivel a lo largo de su trayectoria vital. }\end{array}$ \\
\hline 6 & $\begin{array}{l}\mathrm{PPL}-\text { Percepción de la pre- } \\
\text { paración laboral }\end{array}$ & $\begin{array}{l}\text { Valoró el nivel de preparación que percibieron tener los } \\
\text { deportistas de alto nivel para acceder al mundo laboral de } \\
\text { manera eficaz. }\end{array}$ \\
\hline 7 & SL - Salidas laborales & $\begin{array}{l}\text { Analizó las principales salidas profesionales a las que aspira- } \\
\text { ron los deportistas de alto nivel. }\end{array}$ \\
\hline 8 & TD - Toma de decisiones & $\begin{array}{l}\text { Valoró el modelo y el estilo de toma de decisiones que emplea- } \\
\text { ron los deportistas de alto nivel cuando tenían que tomar una } \\
\text { decisión de carácter relevante en su desarrollo vital. }\end{array}$ \\
\hline
\end{tabular}

Fuente: elaboración propia. 
análisis cualitativo se completó con un estudio de frecuencias, con la finalidad de calcular el número de ocurrencias de las variables tratadas en este trabajo.

\section{Resultados}

Los resultados de este estudio se presentan agrupados en varias dimensiones relacionadas con los objetivos y las variables analizadas.

\subsection{Centralidad y finalidad del trabajo para los deportistas de alto nivel}

Los deportistas entrevistados concedieron una alta importancia al trabajo, puesto que lo consideraron un aspecto fundamental en su desarrollo vital (tabla 3). Este valor que tenía el desarrollo profesional era similar en las diferentes etapas que atravesaban los deportistas en su recorrido vital. Así, los que se encontraban en la fase de iniciación deportiva indicaron que "para mí es importante trabajar, porque, si no trabajas, ¿de qué te mantienes, ¿cómo sobrevives?, ¿cómo mantienes una casa?» (EID4); aquellos que estaban en la etapa de perfeccionamiento deportivo confirmaron que «para mí es importante»; los que se encontraban preparando la retirada manifestaron que «el trabajo es importante, es la fuente de ingresos de la familia» (EPR3), y el grupo de deportistas retirados sugirió que «sin el trabajo ahora mismo no puedo sostener a mi familia y no me puedo sostener a mí mismo» (ERD2).

Ahondando sobre esta cuestión, se comprobó que el trabajo tenía una doble finalidad para los deportistas de alto nivel. Por un lado, era un medio de autorrealización personal y, por otro, un espacio donde adquirir bienes económicos para hacer frente a las situaciones personales (tabla 4). El relato de uno de los deportistas reflejó de manera precisa el sentido que tenía el trabajo cuando indicó que «al final estoy trabajando en un tipo de deporte que está relacionado con mi actividad a nivel profesional, que es la halterofilia, y

Tabla 3. Importancia del trabajo para los deportistas de alto nivel

\begin{tabular}{|c|c|c|}
\hline $\begin{array}{c}\text { Importancia del } \\
\text { trabajo }\end{array}$ & $\begin{array}{l}\text { Número de } \\
\text { referencias }\end{array}$ & Testimonios y relatos de los deportistas de alto nivel \\
\hline Poco importante & 6 & $\begin{array}{l}\text { «Normalmente, un deportista, cuando está en pleno rendi- } \\
\text { miento, muy pocas veces le da poca importancia a lo que } \\
\text { es poder acceder al mercado laboral. En ese momento sola- } \\
\text { mente la meta y la única idea es llegar a lo más alto posible } \\
\text { que puedas y penosamente pocas veces los deportistas } \\
\text { piensan en que el deporte termina algún día.» (ERD2) }\end{array}$ \\
\hline Muy importante & 10 & $\begin{array}{l}\text { «El trabajo es bastante importante, para mí es bastante } \\
\text { importante. No puedo descuidarme de lo que he trabaja- } \\
\text { do porque en el momento en el que, por lo que sea, en } \\
\text { el deporte, una lesión fuerte, una decisión del selecciona- } \\
\text { dor que a mí me deje fuera de la selección española o de } \\
\text { los campeonatos internacionales, yo voy a terminar allí. Es } \\
\text { como un seguro.» (EPD3) }\end{array}$ \\
\hline
\end{tabular}

Fuente: elaboración propia. 
Tabla 4. Finalidad y sentido del trabajo para los deportistas de alto nivel

\begin{tabular}{lcl}
\hline $\begin{array}{l}\text { Finalidad y sentido } \\
\text { del trabajo }\end{array}$ & $\begin{array}{c}\text { Número de } \\
\text { referencias }\end{array}$ & Testimonios y relatos de los deportistas de alto nivel \\
\hline $\begin{array}{l}\text { Autorrealización } \\
\text { personal }\end{array}$ & 8 & $\begin{array}{l}\text { "La verdad es que se trata de una realización personal, aun- } \\
\text { que mi vocación no era la enfermería, pero..., desde luego } \\
\text { le cogí el tranquillo, me gusta y siempre estoy más o menos } \\
\text { estudiando, me reciclo, hago un montón de cursos. (ERD1) }\end{array}$ \\
$\begin{array}{l}\text { Adquisición de bie- } \\
\text { nes económicos }\end{array}$ & 9 & $\begin{array}{l}\text { "Yo creo que para adquirir bienes económicos. Luego con } \\
\text { los bienes económicos ya tendrás más ocasiones de hacer } \\
\text { otras cosas.» (ElD2) }\end{array}$ \\
\hline
\end{tabular}

Fuente: elaboración propia.

a nivel de ganancias también, porque no vivimos solo de realización» (EPD3). Esta argumentación quedó reforzada por los deportistas de alto nivel retirados que estaban trabajando y que concibieron la actividad laboral como un ámbito que «aporta mucha realización personal, porque, bueno, me imagino que si fuese otro tipo de trabajo que, bueno, tuviese que estar en un escritorio o yo que sé, pero como ayudo a la gente y la gente se va muy contenta, la verdad que es un aporte de satisfacción enorme» (ERD3).

\subsection{Importancia de la formación en los procesos de desarrollo profesional}

La información obtenida en las entrevistas puso de manifiesto que los deportistas de alto nivel concedieron una alta importancia a los procesos formativos como un factor que determinó sus posibilidades de éxito en su desarrollo profesional. Algunas de las respuestas de los deportistas reflejaron con claridad la necesidad de tener una formación, dado que «sin estudios no somos nada [...] un futbolista, solo se dedica al fútbol, entonces no tendrá nada que demostrar profesionalmente [...] hay que estudiar» (EPD2). De hecho, la experiencia de los deportistas retirados resaltó la necesidad de «estar formado» (ERD3) como un aspecto clave, básico y fundamental para su desarrollo personal y profesional, dado que, aunque «hayas practicado un deporte de alto nivel, eso no te garantiza que, cuando termines tu carrera, tengas un acceso al mercado laboral, porque no tienes experiencia ninguna, de ningún otro tipo de trabajo que no sea del mundo del deporte» (ERD2). Efectivamente, uno de los deportistas retirados manifestó la importancia de ir preparándose para el futuro profesional cuando sugirió:

Si tú me preguntas ahora [...] si yo pudiera tener la oportunidad de regresar atrás a tener los dieciocho años, diecisiete años $[\ldots]$, y tengo que volver a tomar una decisión sobre qué es lo que yo quiero hacer, yo pienso que hubiese preferido estudiar, porque tienes más garantías si tú estudias, si tú te preparas en una carrera universitaria, que dedicarte al mundo del deporte profesional, el deporte de alto nivel. (ERD2)

Pese a la importancia que le concedieron los deportistas de alto nivel a la formación como un factor determinante para su futuro desarrollo profesional, 
también eran conscientes de las dificultades que existían para mantener una relación efectiva entre la práctica deportiva y la formación académica. Por ello, los deportistas de alto nivel indicaron que «si llegas a jugar en el máximo nivel es muy difícil compaginar el deporte con los estudios» (ERD2). Uno de los deportistas entrevistados señaló que «al principio pues sí que podía compaginar mejor el fútbol [...] con el tema del estudio, pero cada año, al ser más exigente el nivel, pues también me requiere más tiempo y además también se vuelve más exigente el nivel de estudios» (EPD4). Como consecuencia, muchas de las personas vieron limitadas sus posibilidades de rendimiento en las actividades deportivas y académicas, lo que les llevó a situaciones en las que no pudieron dedicar el «cien por cien a la carrera ni el cien por cien al deporte, y entonces empecé con Biología el primer año y lo acabé dejando, porque el deporte me sacaba muchas horas» (EPD1). Por ello, las personas encuestadas coincidieron al afirmar que «es difícil estudiar y a la vez entrenar» (EID3).

El principal hándicap que encontraron los deportistas de alto nivel para llevar a término este proceso de compatibilización entre estudios y deporte fue la falta de tiempo. En este sentido, reflejaron que había que estar dispuestos a «sacrificar tiempo para otras cosas para dedicarlo al deporte, ya sea con la vida laboral o los estudios o la vida familiar» (EPR2). Incluso algunos de ellos manifestaron que era posible mantener un cierto equilibro entre estas dos actividades priorizándolas con un peso específico de «un sesenta o un setenta por ciento de experiencia de natación y un cuarenta o un treinta por ciento de formación» (EPD2). De cualquier manera, los deportistas afirmaron que, para salvaguardar estos dos procesos, era importante aprender y dominar adecuadamente la competencia de "gestionar el tiempo» (EPR2) y «organizarse bien» (EID2). Entendieron que solo de esta manera se podía «entrenar y competir a la vez» (EID3).

\subsection{Conocimiento sobre el mercado laboral, itinerarios profesionales y preparación para el mundo profesional}

En general, los deportistas de alto nivel tenían información sobre tres aspectos básicos del mercado laboral: condiciones de acceso a los puestos de trabajo (I), características específicas de los empleos (II) y salidas profesionales a las que podían optar (III). Respecto al primero de ellos, indicaron que conocían «los requisitos que me piden para acceder al puesto de trabajo» (EPD4), en relación con el segundo, tenían información relacionada con «el ámbito que toca, que es todo de ayudas sociales» (EPD1) y, finalmente, conocían «todo el abanico de posibilidades al que se puede optar» (EPD4).

Una importante información que arrojó el análisis de las entrevistas fue la definición de los itinerarios académicos y profesionales de los deportistas de alto nivel. Concretamente, una de las personas entrevistadas afirmó que «tenía bastante claro lo que había de hacer para luego ser un entrenador y seguir con esa faceta, y lo veía también en los entrenadores que yo había tenido a lo largo de mi carrera y siempre me había gustado y eso, y sabía cómo prepararme para 
dar ese paso» (EPR3). A modo de ejemplo, uno de los deportistas relató los pasos que siguió en su trayectoria personal para llegar a ser entrenador profesional. De manera específica fue

[...] sacando los títulos de entrenador [...], primero entrenador territorial, entrenador local, luego entrenador nacional y a la vez entrenaba a equipos de cerca de mi casa o equipos de categorías inferiores de donde yo jugaba [...] tenía en mente que, después de acabar de jugar, mi otra fase con el deporte podía ser la de entrenador y, durante mi carrera como deportista, me preparé para eso. (EPR3)

Además de tener claro el itinerario a seguir para lograr sus metas profesionales, los deportistas eran conscientes de las dificultades que podían encontrar por el camino. En este sentido, uno de los testimonios aportados por los deportistas reflejó de manera precisa esta situación cuando manifestó:

[aunque] lo tenga claro no significa que lo consiga. Es decir, al final es complicado ser un profesional del deporte, pero si sabemos cuáles son los pasos a seguir para llegar a conseguirlo, llegar a serlo, igual que en el tema de los estudios, para ser un profesional de lo que estoy estudiando, pues también conozco los pasos y el sacrificio que hay que hacer para ambas cosas, así que si no es el deporte, si no consigo alcanzar ser un profesional del deporte, pues tengo claro que puedo alcanzar ser un profesional de mis estudios, de lo que estoy estudiando, pero en principio mi objetivo es ser profesional de ambas cosas. (EPD4)

Otro de los aspectos que se valoró en el desarrollo de las entrevistas fue la percepción que tenían los deportistas de alto nivel de estar preparados para adaptarse de manera eficaz al mundo profesional. De hecho, del discurso analizado se pudieron extraer dos ideas básicas. La primera de ellas sugirió que los deportistas, aun cuando percibieron no estar preparados para afrontar con éxito el acceso y la adaptación al mercado laboral, encontraron en la formación una estrategia básica para cubrir sus carencias y sus debilidades. En este sentido, indicaron que «la formación que estoy recibiendo ahora mismo pues el día de mañana sé que si las cosas van bien y lo consigo sacar, pues sí que llegaré con la preparación que requiere un puesto de trabajo» (EPD4). En cuanto a la segunda, los entrevistados eran conscientes de que, aunque tuvieran una formación académica adecuada para desempeñar un trabajo cualificado, era importante participar en experiencias profesionales para mejorar su capacitación laboral. En concreto, los deportistas señalaron lo siguiente:

[...] de un día para otro no pasas de ser jugador a entrenador de la selección española en el torneo más importante del mundo, sino que primero, pues, pasas a entrenar a los juveniles de la selección navarra, de ahí pasé a los juveniles de la selección española, o sea, fui dando pasos hacia delante y fui cogiendo experiencia hasta que ya llegué a la selección española absoluta y me sentía preparado para hacer el trabajo. (EPR3) 


\subsection{Oportunidades laborales, metas profesionales y de toma de decisiones}

En general, una amplia mayoría de deportistas de alto nivel $(n=13)$ había tenido la oportunidad de participar en experiencias laborales. En un análisis más preciso se comprobó que, a medida que el deportista se aproximaba al momento de la retirada, mayores eran las oportunidades profesionales que tenía. De hecho, en la fase de iniciación ninguno de los deportistas entrevistados vivió experiencias de este tipo, mientras que en las fases de perfeccionamiento, preparación de la retirada y retirados tuvieron contacto con el mundo profesional (figura 1).

Ahondando sobre esta cuestión, se comprobó que muchas de estas oportunidades profesionales no necesariamente guardaban relación con el mundo deportivo (tabla 5). Aproximadamente uno de cada dos deportistas de alto nivel cuando pensó en su futuro laboral afirmó que «solo veo objetivos profesionales» (EPD1), mientras que el resto proyectó su trayectoria de trabajo hacia otras salidas que no tenían que ver con el ámbito deportivo. Esta situación se comprobó mediante un análisis más preciso de la información obtenida en las entrevistas, donde se extrajeron algunos de los intereses profesionales de los deportistas de alto nivel, como fueron ser «veterinario» (EID1), «futbolista» (EPD4), «entrenador de natación» (EPD2), «policía nacional» (EPD3) o «psicóloga» (EID2).

Figura 1. Oportunidades laborales de los deportistas de alto nivel en función de su etapa profesional

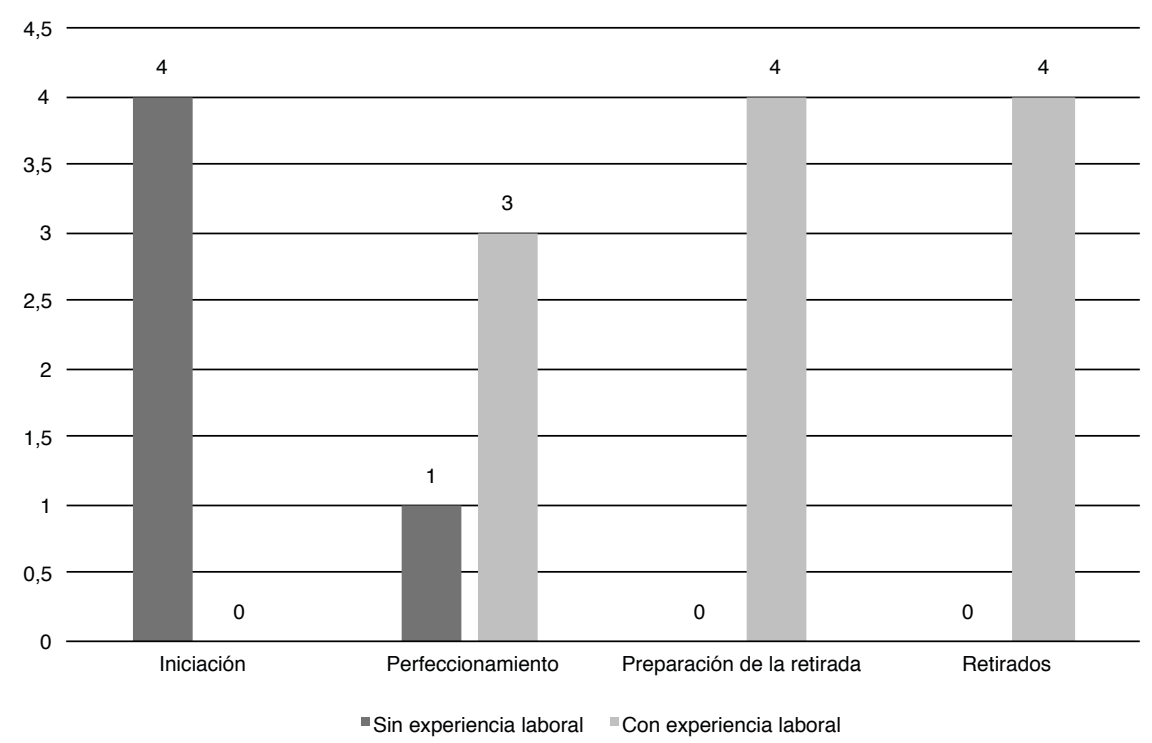

Fuente: elaboración propia. 
Tabla 5. Intereses y metas profesionales de los deportistas de alto nivel

\begin{tabular}{lcc}
\hline $\begin{array}{l}\text { Metas e intereses } \\
\text { profesionales }\end{array}$ & $\begin{array}{c}\text { Número de } \\
\text { referencias }\end{array}$ & Testimonios y relatos de los deportistas de alto nivel \\
\hline $\begin{array}{l}\text { Vinculados al } \\
\text { mundo deportivo }\end{array}$ & 6 & $\begin{array}{l}\text { "Lo natural, es decir, he sido deportista y he continuado } \\
\text { siéndolo, pues sería ser entrenador, si pudiera percibir una } \\
\text { remuneración por ello y me pudiera dedicar, que me pudie- } \\
\text { ra dedicar exclusivamente al entrenamiento, es decir, o a } \\
\text { cualquier otra actividad que me permitiese seguir vinculado } \\
\text { al deporte que he estado practicando, el atletismo en este } \\
\text { caso, de una forma remunerada.» (EPR1) }\end{array}$ \\
$\begin{array}{l}\text { Vinculados a otros } \\
\text { ámbitos profesio- } \\
\text { nales }\end{array} \quad 7 \quad \begin{array}{l}\text { "A mí me gustaría mucho trabajar en la policía nacional, igual } \\
\text { que de bombero, lo que pasa que bombero se me escapa } \\
\text { mucho más, y tienes que estar muchísimos años dedicándo- } \\
\text { te a ello y es complicado, a parte de que el límite de plazas } \\
\text { es bastante inferior al de policía.» (EPD3) }\end{array}$ \\
\hline
\end{tabular}

Fuente: elaboración propia.

En las entrevistas se comprobó que los deportistas de alto nivel valoraron la importancia que tenían algunas personas en el proceso de definición de sus intereses profesionales. En este sentido, consideraron que siempre hay "personas que te orientan o te ayudan a determinadas cosas o te facilitan cierto desarrollo» (EPR2). Efectivamente, uno de los entrevistados apuntó la incidencia que tuvo «mi padre, ya que ha influido mucho en todo lo que me gusta a mí hacer. Mi padre quiso ser bombero en su momento y, bueno, él me inspiró» (EID3).

En cuanto a los procesos de toma de decisiones, los resultados obtenidos confirmaron que los deportistas de alto nivel utilizaron un sistema basado en la razón. En este ejercicio de elección valoraron «que me satisfaga de alguna manera lo que estoy haciendo, el que me haga feliz, el que me encuentre plenamente satisfecho y a continuación tomo la decisión, o sea, no voy a tomar nunca un camino donde verdaderamente no me sienta bien» (EPR4). Además, entendieron que, para tomar una decisión de manera eficaz, «siempre debes tener en cuenta qué es lo que quieres, cuál es tu objetivo y, pues, a partir de ahí adaptarte a las diferentes posibilidades que tengas para llegar a conseguirlo (EPR3).

\section{Conclusiones}

En este trabajo de investigación se ha realizado un análisis sobre las características del desarrollo profesional y del proyecto personal y de vida de los deportistas de alto nivel del contexto español. La principal conclusión que se puede extraer de los hallazgos obtenidos es que son conscientes de la importancia que tiene su futuro desarrollo laboral. De hecho, los relatos aportados en las entrevistas realizadas coinciden con estudios previos (Alonso, 2004; Sverko, 2001; MOW, 1987), al sugerir que el trabajo ocupa un lugar importante y constituye una meta básica y central en la trayectoria personal del deportista. De los resultados obtenidos se desprende también que, para los que 
participan en competiciones de alto nivel, el trabajo puede tener una doble finalidad. Mientras unos consideran que el sentido básico de la actividad profesional es la autorrealización personal, otros afirman que se trata de un espacio para obtener bienes económicos (Ibáñez y Mudarra, 2007; Salanova, Osca, Peiró y Sarcerni, 1991).

El estudio realizado demuestra que la actividad formativa y la práctica de su disciplina constituyen dos importantes factores en los procesos de desarrollo profesional de los deportistas de alto nivel (González y Bedoya, 2008; Morales, 2009; Puig y Vilanova, 2006; Torregrosa, Sánchez y Cruz, 2004; Sarramona, 2002). Tal como apuntan McKnight et al. (2009), la formación contribuye a conseguir el logro de las metas profesionales y mejora los procesos de transición y adaptación al mundo laboral y, por ello, debe ocupar un lugar significativo en la vida de los deportistas. Sin embargo, armonizar la actividad formativa y la práctica de su disciplina podría verse comprometido por la falta de habilidades y competencias para gestionar el tiempo de una manera eficaz. Coincidiendo con los resultados de otros trabajos (López et al., 2015; Álvarez y López, 2012; Puig y Vilanova, 2006; Nuviala y Nuviala, 2005) que han analizado las dificultades que encuentra el deportista en este proceso de compatibilización, la falta de tiempo es una variable que puede determinar y complicar las posibilidades de simultanear el deporte con los estudios. Por ello, y ante esta situación, los deportistas son concluyentes al afirmar que solo es posible mantener esta actividad dual si se domina la competencia de planificación y de gestión del tiempo.

Los datos presentados en el estudio reflejan que el conocimiento que tienen los deportistas de alto nivel sobre el mercado de trabajo gira en torno a tres aspectos básicos: condiciones de acceso a los puestos de trabajo (I), características específicas de los empleos (II) y salidas profesionales a las que pueden optar (III). De acuerdo con los planteamientos de Lent et al. (2000), Lent, Hill y Hofman (2003) y Romero (2004), esta información permite a los deportistas de alto nivel diseñar un itinerario y construir un proyecto profesional y vital en consonancia con su contexto sociolaboral y con sus expectativas personales. Profundizando en este aspecto, se comprobó que uno de cada dos deportistas proyecta su escenario profesional y su itinerario personal hacia un empleo que no necesariamente guarda relación con el ámbito deportivo.

La información sobre el proceso de toma de decisiones pone de manifiesto que los deportistas de alto nivel utilizan un sistema basado en la razón. Concretamente, en este ejercicio de elección, valoran lo que es deseable frente a lo que es probable y analizan de manera precisa las consecuencias de las distintas alternativas a las que pueden optar. Se trata, de acuerdo con Lent, Brown y Hackett (1994) y Lent, Brown y Hackett (2000), de un proceso en el que entran en juego los conceptos de autoeficacia (lo que soy capaz de hacer) y las expectativas de resultado (lo que espero conseguir).

Finalmente, y a partir de los hallazgos de este estudio, es importante reflexionar sobre algunas acciones de interés que pueden servir para mejorar 
los procesos de desarrollo profesional de los deportistas de alto nivel desde la práctica educativa y orientadora:

- Diseñar herramientas y estrategias educativas y orientadoras que permitan a los deportistas de alto nivel armonizar de una manera efectiva la práctica de su disciplina con su formación académica, de tal manera que, a lo largo de su existencia, puedan ir definiendo un proyecto profesional y vital que los guíe y los oriente en su trayectoria personal. En este sentido, sería interesante que cada deportista contara con un tutor personal que le ayudara en la mediación del conflicto de intereses entre estudios y deporte, le facilitara la construcción de proyectos académicos y profesionales, le ofreciera estrategias para conocerse a sí mismo y al entorno, le brindara la oportunidad de desarrollar competencias y habilidades de interés para su desarrollo profesional, etc.

- Aunar esfuerzos y ofrecer espacios de diálogo entre las estructuras académicas (Ministerio de Educación, Consejería de Educación, centros educativos, etc.) y las deportivas (Consejo Superior de Deportes, Consejería de Deportes, clubes deportivos, etc.), para que los deportistas de alto nivel cuenten con el apoyo de estos dos procesos que tan importantes son para el desarrollo profesional.

- Sensibilizar a los deportistas sobre la importancia que tiene tomar decisiones asentadas en la realidad, es decir, elegir aquellas alternativas formativas y/o profesionales que mejor se ajusten a sus potencialidades (autoeficacia) y a sus posibilidades (expectativas de resultado).

- Ayudar a los deportistas de alto nivel para que conozcan de manera precisa las condiciones laborales y sociales, de tal manera que la definición de su identidad vocacional y los procesos de toma de decisiones sean realistas y estén acordes al contexto situacional en el que se desenvuelve el deportista.

- Ofrecer al deportista la oportunidad de participar en experiencias preprofesionales que le permitan conocer de primera mano el mundo del trabajo.

\section{Referencias bibliográficas}

Alfermann, D.; Stambulova, D. y Zemaityte, A. (2004). Reactions to sport career termination: A cross - national comparison of German, Lithuanian and Russian athletes. Psychology of Sport and Exercise, 5, 61-75. <https://doi.org/10.1016/s1469-0292(02)00050-x>

Alonso, M. (2004). Centralidad del trabajo y metas en el trabajo. Revista de Educación, 335, 319-344.

Álvarez, P. y López, D. (2012). Armonización entre proceso de aprendizaje y práctica deportiva en universitarios deportistas de alto nivel. Cultura, Ciencia y Deporte, 7(21), 201-212.

<https://doi.org/10.12800/ccd.v7i21.85>

BAILLIE, P.H.F. y DANISH, S. (1992). Understanding the career transition of athletes. The Sport Psychologist, 6, 77-98. <https://doi.org/10.1123/tsp.6.1.77> 
Bruner, M.W.; Munroe-Chandler, K.J. y Spink, K. (2008). Entry into Elite Sport: A preliminary investigation into the transition experiences of Rookie Athletes. Journal of Applied Sport Psychology, 20, 236-252. <https://doi.org/10.1080/10413200701867745>

Carlin, M. y Garcés, E. (2012). Transición de carrera deportiva a un nuevo contexto vital: Perspectivas actuales. Cuadernos de Psicología del Deporte, 12(1), 103-110. <https://doi.org/10.4321/s1578-84232012000100009>

Cecic-Erpic, S.; Wylleman, P. y Zupancic, M. (2004). The effect of athletic and non-athletic factor son the sports career termination process. Psychology of Sport and Exercise, 5, 45-59. <https://doi.org/10.1016/s1469-0292(02)00046-8>

Corbeta, P. (2003). Metodología y técnicas de investigación social. Madrid: McGraw- Hill.

Echeverría, B. (1997). Inserción sociolaboral. Revista de Investigación Educativa, $15(2), 85-115$.

Fernández, A.; Stephan, Y. y Fouquereau, E. (2006). Assessing reasons for sport career termination: Development of the Athletes' Retirement Decision Inventory (ARDI). Psychology of Sport and Excercise, 7, 407-421. <https://doi.org/10.1016/j.psychsport.2005.11.001>

Franklin, C. y Ballau, M. (2005). Reliability and validity in qualitative research. En R.M. Grinell y Y.A. Unrau (eds.). Social work: Research and evaluation. Quantitative and qualitative approaches (pp. 438-449). Nueva York: Oxford University Press.

Garcés, E.J. y CARLIN, M. (2011). El proceso de retirada y ajuste vital en deportistas de alto rendimiento. Sevilla: Wanceulen Editorial Deportiva.

González, M.D. y Bedoya, J. (2008). Después del deporte, ¿qué?: Análisis psicológico de la retirada deportiva. Revista de Psicología del Deporte, 17(1), 61-69.

González, M.D. y Torregrosa, M. (2009). Análisis de la retirada de la competición de élite: Antecedentes, transición y consecuencias. Revista Iberoamericana de Psicología del Ejercicio y del Deporte, 4(1), 93-104.

Guichard, J. (1995). Psychopedagogie du projet d'avenir et normativité. En ADAPT (ed.). Projets des jeunes: Une question d'identité (pp. 9-40). París: ADAPT.

Hernández, R.; Fernández-Collado, C. y Baptista, P. (2006). Metodología de la Investigación. $4 .^{\mathrm{a}}$ ed. México: McGraw-Hill.

IbáÑez, P. y Mudarra, M.J. (2007). Integración sociolaboral. Madrid: Dykinson.

JodaI, K. y Nogawa, H. (2012). A study of professional athlete career transition: And overview of the literature. Football Science, 9, 50-61.

Kerr, G. y Dacyshyn, A. (2000). The retirement experiences of elite, female gymnasts. Journal of Applied Sport Psychology, 12, 115-133. <https://doi.org/10.1080/10413200008404218>

Lent, R.W.; Brown, S. y Hackett, G. (1994). Toward a unifying social cognitive theory of career and academic interest, choice, and performance. Journal of Vocational Behavior, 45, 79-122. <https://doi.org/10.1006/jvbe.1994.1027>

- (2000). Contextual supports and barriers to career choice: A social cognitive analysis. Journal of Counseling Psychology, 47(1), 36-49. <https://doi.org/10.1037//0022-0167.47.1.36>

Lent, R.W.; Hill, C.E. y Hoffman, M.A. (2003). Development and validation of the counselor activity self - efficacy scales. Journal of Counseling Psychology, 50(1), 97-108. <https://doi.org/10.1037//0022-0167.50.1.97> 
López, C.; Barriopedro, M.; Conde, E.; Sánchez, J.; Ubago, E. y Gallardo, L. (2015). Análisis de las barreras percibidas por los deportistas de élite españoles para acceder a los estudios. Cuadernos de Psicología del Deporte, 15(1), 265-274. <https://doi.org/10.4321/s1578-84232015000100025>

Martínez, P. (2008). Orientación profesional para la transición. En B. Echeverría, S. Isus, M.P. Martínez y L. SARAsola (2008). Orientación profesional (pp. 223300). Barcelona: UOC.

McKnight, K.; Bernes, K.; Gunn, T.; Chorney, D.; Orr, D. y Bardick, A. (2009). Life after sport: Athletic career transition and transferable skills. Journal of Excellence, 13, 63-77.

McMillan, E. y Schumacher, S. (2005). Investigación educativa. 5. a ed. Madrid: Pearson. Mertens, D.M. (2005). Research and evaluation in Education and Psychology: Integrating diversity with quantitative, qualitative and mixed methods. Thousand Oaks: Sage.

Morales, J.A. (2009). Formación a lo largo de la vida: Realidades y retos. En J. TEJADA (coord.). Estrategias de innovación en la formación para el trabajo (pp. 355374). Madrid: Tornapunta Ediciones.

MOW (1987). Meaning of working: An International View. Londres: Academic Press.

North, J. y Lavallee, D. (2004). An investigation of potential users of career transition services in the United Kingdom. Psychology of Sport and Exercise, 5, 77-84. $<$ https://doi.org/10.1016/s1469-0292(02)00051-1>

Nuviala, A. y Nuviala, R. (2005). Abandono y continuidad de la práctica deportiva escolar organizada desde la perspectiva de los técnicos de una comarca aragonesa. Revista Internacional de Medicina y Ciencias de la Actividad Física y el Deporte, 5(20), 295-307.

Patton, W. y McMahon, M. (2006). The systems theory framework of career development and counselling: Connecting theory and practice. International Journal for the Advancement of Counselling, 28(2), 153-166. <https://doi.org/10.1007/s10447-005-9010-1>

Pearson, R.E. y Petitpas, A.J. (1990). Transitions of athletes: Developmental and preventive perspectives. Journal of Counseling and Development, 69, 7-10. <https://doi.org/10.1002/j.1556-6676.1990.tb01445.x>

Puig, N. y Vilanova, A. (2006). Deportistas olímpicos y estrategias de inserción laboral: Propuesta teórica, método y avance de resultados. Revista Internacional de Sociología, LXIV(44), 63-83. <https://doi.org/10.3989/ris.2006.i44.28>

Rincón, D. del; Latorre, A.; Arnal, J. y Sans, A. (1995). Técnicas de investigación en ciencias sociales. Madrid: Dykinson.

Rodríguez, M.L. (1999). La preparación para el trabajo: El proyecto profesional. Revista d'Orientació, XII, 5-22.

Rodríguez, M.L.; Álvarez, M.; Figuera, M.P. y Rodríguez, S. (2009). Estudios universitarios, proyecto profesional y mundo del trabajo. Barcelona: Universidad de Barcelona. Servicio de Publicaciones.

Romero, S. (2004). Aprender a construir proyectos profesionales y vitales. Revista Española de Orientación y Psicopedagogía, 15(2), 337-354.

Romero, S.; Seco, M. y Lugo, M. (2015). Orientar desde el ser: Algunas aportaciones del programa orient@cual para la elaboración del proyecto profesional y vital del alumnado de PCPI y CFGM. Revista Electrónica Interuniversitaria de Formación del Profesorado, 18(2), 75-89.

<https://doi.org/10.6018/reifop.18.2.219121> 
Salanova, M.; Osca, M.; Peiró, J.M. y Sarcerni, M. (1991). Significado del trabajo en los jóvenes en la transición e incorporación al mercado laboral. Revista de Psicología General y Aplicada, 44(1), 113-125.

SARASON, I.G. y SARASON, B.R. (1986). Experimentally provided social support. Journal of Personality and Social Psychology, 50, 1222-1225. <https://doi.org/10.1037//0022-3514.50.6.1222>

Sarramona, J. (2002). La formación continua laboral. Madrid: Biblioteca Nueva.

Surujlal, J. y ZyL, Y. (2014). Understanding the dynamics of Sport: Career transition of Olympic Athletes. Mediterranean Journal of Social Sciences, 5(20), 477-484. <https://doi.org/10.5901/mjss.2014.v5n20p477>

Sverko, B. (2001). Life Roles and Values in International Perspective: Supers Contribution through the Work Importance Study. International Journal for Educational and Vocational Guidance, 1, 121-130.

Taylor, J. y Ogilvie, B. (1994). A conceptual model of adaptation to retirement among athletes. Journal of Applied Sport Psychology, 6, 1-20. <https://doi.org/10.1080/10413209408406462>

Torregrosa, M.; Sánchez, X. y Cruz, J. (2004). El papel del psicólogo del deporte en el asesoramiento académico-vocacional del deportista de élite. Revista de Psicología del Deporte, 13(2), 2156-2228.

Warriner, K. y Lavallee, D. (2008). The retirement experiences of Elite Female Gymnasts: Self Identity and the Physical Self. Journal of Applied Sport Psychology, 20, 301-317. <https://doi.org/10.1080/10413200801998564>

Wong, H. (2010). The transition process from competitive sport to retirement: Future engagement in physical activity and involvement in figure skating. Master's Thesis in Behavioral Psychology and Kinesiology. Toronto, ON. Canadá: The University of York.

Wylleman, P. y Lavalle, D. (2004). A developmental perspective on transitions faced by athletes. En M. Weiss (ed.). Developmental sport and excercise psychology: $A$ lifespan perspective (pp. 507-527). Morgantown, NTW: Fitness Information Technology.

Wylleman, P.; Lavallee, D. y Alfermann, D. (1999). Career transitions in competitive sports. Biel, Suiza: FEPSAC. 